\title{
Detection of thermal bridges in insulating stratified media with thermography-a 2D transient direct model suitable to implement a Total Least Squares estimation method.
}

\author{
by M. Bamford*, J.C. Batsale*, D. Mourand* and H. Bendada** \\ *TREFLE-ENSAM, UMR 8508 CNRS, 33405 Talence cedex, France \\ ${ }^{*}$ LVSN, Laval University, Pavillon Adrien-Pouliot, G1K-7P4 - Quebec, Canada
}

\begin{abstract}
The aim of this work is to conceive a thermal NDE method in order to detect source terms placed under thin diffusive plates, with an extension to thermal bridges in insulating stratified media. Contrarily to the detection of delaminating in composite samples, such situation induces strong 2D transient effects which force us to consider the spatial correlation between the pixels. In order to avoid a heavy inversion process, a suitable approximation of the exact analytical model of heat diffusion is developed, in order to estimate the lateral size of the source term. The interesting feature of this method is that robust estimations are processed with no prior knowledge of the measurement noise variance. Experimental results illustrate the method.
\end{abstract}

\section{Introduction}

Infrared thermography provides a tool for drawing thermal properties mappings. It is thus often used in NDE of laminates. Some possible applications are to detect delaminated areas in stratified media [1], to find in-plane defects on thin plates [2], to find cracks in semi infinite media [3], or even to locate thermal bridges in insulating stratified media [4].

In the case of thermal bridges between two plates, the difficulty in the estimation of the size and location of the contact is the same as for any source term under a plate: 2 dimensional heat transfers in the front face of the device induce a "blurring" of the initial shape of the source term. An inversion can be performed with the knowledge of the heat transfer model to trace the original pattern of the heat source. Such an inversion can be performed by analysing the relations between spatial and temporal derivatives of the front face temperature field. This study can be done in a sequential way as proposed in $[5,6]$. In a similar fashion the Total Least Squares (TLS) method $[7,8]$ is very suitable because it estimates robustly a source term $Q(x, y)$ placed under the front face at each pixel, and is implemented in an autoregressive way. The computation of the $2 \mathrm{D}$ source terms field is then available. Yet this method requires a linear formulation of the problem. To this aim a simplified model is developed. Contrarily to [1], where defects have to be of small lateral size at a known location, no constraining hypotheses are required here.

Such situation allows studying the exact dimension of very small diameter heat sources through opaque layers. In such cases, the spatial camera resolution or the considered layer thickness are limiting the estimation of the contact real size. Nevertheless, a local contact with a heat source can be estimated and constitutes a first approach in order to study very small contacts. 


\section{http://dx.doi.org/10.21611/qirt.2006.059}

In this study, a simplified model of heat transfers in stratified media with thermal bridges is presented. A parallel is drawn with the case of a resistive wire placed under a diffusive thin plate. The same model is used to implement the TLS estimator in the two cases.

\section{3D Direct model}

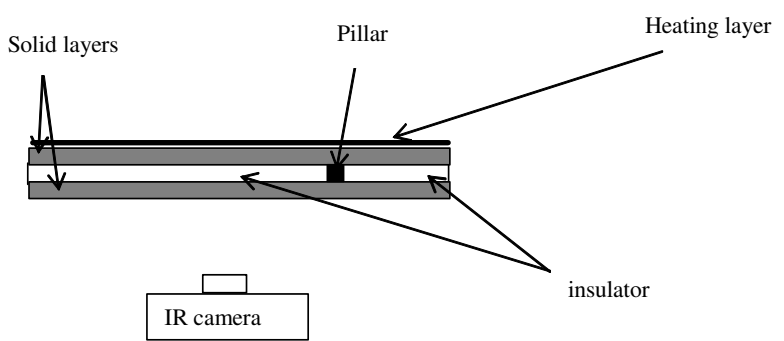

Fig. 1. Scheme of the stratified medium with thermal bridges

In a classical way, a constant heat flux is applied on the rear face by means of a heating layer. The thermal scene is shot with an infrared camera on the front face. The thermal insulation is considered as a purely resistive layer of null thickness. The heat transfer is then governed by the following equations:

$$
\begin{aligned}
& \frac{\partial^{2} T}{\partial x^{2}}+\frac{\partial^{2} T}{\partial y^{2}}+\frac{\partial^{2} T}{\partial z^{2}}=\frac{1}{a} \cdot \frac{\partial T}{\partial t} \\
& T(x, y, z, t=0)=0 \\
& -\lambda \cdot \frac{\partial T}{\partial z}=Q \cdot H(t) \quad \text { at } z=0 \\
& -\lambda \cdot \frac{\partial T}{\partial z}=\frac{1}{R(x, y)} \cdot\left[T^{\text {sup }}(x, y, e, t)-T^{\text {inf }}(x, y, e, t)\right] \quad \text { at } z=e \\
& -\lambda \cdot \frac{\partial T}{\partial z}=0 \quad \text { at } z=2 e \\
& \frac{\partial T}{\partial x}=0 \quad \text { at } x=0 \text { and } x=l ; \frac{\partial T}{\partial y}=0 \quad \text { at } y=0 \text { and } y=L
\end{aligned}
$$

This 3D problem has been studied with the quadrupole approach $[2,3,4,9,10,11]$. The Fourier integral transform versus $x$ and $y$ space variables and the Laplace transform versus time are used to obtain: 
$\theta(n, m, z, p)=\int_{t=0}^{\infty} \int_{x=0}^{l} \int_{y=0}^{L} \cos \left(\alpha_{n} \cdot x\right) \cos \left(\beta_{m} \cdot y\right) \exp (-p \cdot t) \cdot T(x, y, z, t) d t d x d y$

With $\alpha_{n}=\frac{n \pi}{l} ; \beta_{m}=\frac{m \pi}{L}$. Using a vector notation such as $\mathbf{T}(z, p)=\left[\theta(0,0, z, p), \ldots, \theta\left(\alpha_{n}, \beta_{m}, z, p\right), \ldots\right] n \in[0, l-1] ; m \in[0, L-1] \quad$ it yields that:

$$
\left[\begin{array}{c}
\mathbf{T}(0, p) \\
\mathbf{Q} / p
\end{array}\right]=\left[\begin{array}{ll}
\mathbf{A} & \mathbf{B} \\
\mathbf{C} & \mathbf{D}
\end{array}\right]\left[\begin{array}{ll}
\mathbf{I} & \mathbf{R} \\
\mathbf{0} & \mathbf{I}
\end{array}\right]\left[\begin{array}{ll}
\mathbf{A} & \mathbf{B} \\
\mathbf{C} & \mathbf{D}
\end{array}\right]\left[\begin{array}{c}
\mathbf{T}(2 e, p) \\
\mathbf{0}
\end{array}\right]
$$

The terms of the transfer matrix related to the transfer inside the conductive layers are purely diagonal:

$$
\begin{aligned}
& \mathbf{A}=\operatorname{diag}\left[\ldots, \operatorname{ch}\left(e \sqrt{\frac{p}{a}+\alpha_{n}^{2}+\beta_{m}^{2}}\right), \ldots\right]=\mathbf{D}, \\
& \mathbf{B}=\operatorname{diag}\left[\ldots, \operatorname{sh}\left(e \sqrt{\frac{p}{a}+\alpha_{n}^{2}+\beta_{m}^{2}}\right) / \lambda \sqrt{\frac{p}{a}+\alpha_{n}^{2}+\beta_{m}^{2}}, \ldots\right], \\
& \mathbf{C}=\operatorname{diag}\left[\ldots, \operatorname{sh}\left(e \sqrt{\frac{p}{a}+\alpha_{n}^{2}+\beta_{m}^{2}}\right) \cdot\left(\lambda \sqrt{\frac{p}{a}+\alpha_{n}^{2}+\beta_{m}^{2}}\right), \ldots\right],
\end{aligned}
$$

The heat flux at $z=0$ is assumed to be uniform and so the Fourier transform vector has only one component at the null spatial frequency, such as:

$$
\mathbf{Q}=[Q l L, 0, \ldots, 0, \ldots]^{T}
$$

$\mathbf{I}$ is the identity matrix and $\mathbf{R}$ is a full matrix traducing the spatially non uniform transfer at $z=e$. The form of this matrix was discussed in $[10,11]$. The temperature vector in transformed space at $z=2 e$ is then:

$$
\mathbf{T}(z=2 e, p)=\left(\mathbf{A}^{2}+\mathbf{B C}+\mathbf{C R C}\right)^{-1} \mathbf{Q} / p
$$

With this expression the formally exact solution is known. Yet the matrices to be inverted are large, and the numerical implementation is thus computationally heavy. Yet a first order approximation can be drawn provided the amplitude of $\mathbf{R}$ is large enough:

$$
\mathbf{T}(z=2 e, p)=(\mathbf{C R C})^{-1} \mathbf{Q} / p
$$




\section{http://dx.doi.org/10.21611/qirt.2006.059}

At long times $(p \rightarrow 0)$, and provided the conductive layers are isothermal following the thickness direction. The following relation holds in the Laplace-Fourier space:

$$
\mathbf{C} \approx \operatorname{diag}\left[\ldots, \lambda e\left(\frac{p}{a}+\alpha_{n}^{2}+\beta_{m}^{2}\right), \ldots\right]
$$

It yields:

$$
\boldsymbol{\operatorname { R C T }}(z=2 e, p)=\operatorname{diag}\left[\ldots, \frac{1}{\lambda e\left(\frac{p}{a}+\alpha_{n}^{2}+\beta_{m}^{2}\right)}, \ldots\right][Q l L, 0, \ldots, 0, \ldots]^{T}
$$

Thus

$$
\mathbf{R} \cdot \operatorname{diag}\left[\ldots, \lambda e\left(\frac{p}{a}+\alpha_{n}^{2}+\beta_{m}^{2}\right), \ldots\right] \mathbf{T}(z=2 e, p)=\left[\frac{a}{\lambda e p} Q l L, 0, \ldots, 0, \ldots\right]^{T}
$$

So that when applying the inverse Fourier and Laplace transforms the following relation stands:

$$
R(x, y) \cdot\left[\frac{\partial T}{\partial t}-a \cdot\left(\frac{\partial^{2} T}{\partial x^{2}}+\frac{\partial^{2} T}{\partial y^{2}}\right)\right](x, y, z=2 e, t)=\frac{Q}{(\rho c \cdot e)^{2}} \cdot t
$$

In the case of a resistive wire placed under a diffusive thin plate, the problem is very similar

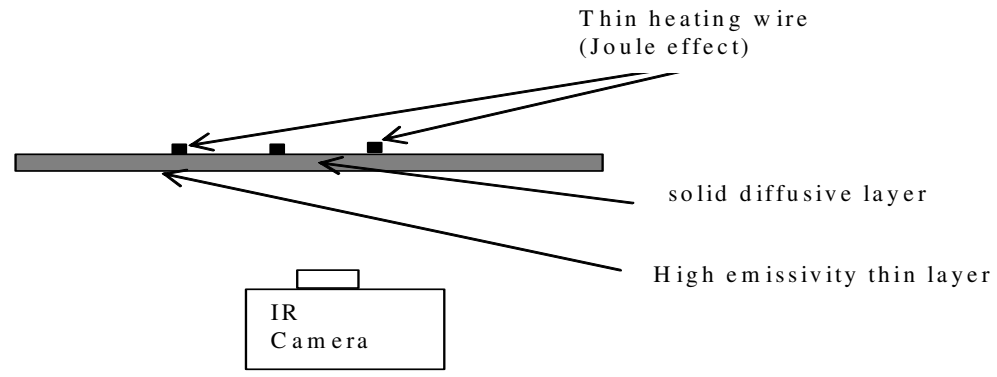

Fig. 2. Scheme of the resistive wire placed under a diffusive plate 
The quadrupole formulation is even simpler in this case since

$$
\left[\begin{array}{c}
\mathbf{T}(0, p) \\
\mathbf{Q} / p
\end{array}\right]=\left[\begin{array}{cc}
\mathbf{I} & \mathbf{R}^{\prime} \\
0 & \mathbf{I}
\end{array}\right]\left[\begin{array}{cc}
\mathbf{I} & 0 \\
\mathbf{C}^{\prime} & \mathbf{I}
\end{array}\right]\left[\begin{array}{ll}
\mathbf{A} & \mathbf{B} \\
\mathbf{C} & \mathbf{D}
\end{array}\right]\left[\begin{array}{c}
\mathbf{T}(e, p) \\
\mathbf{0}
\end{array}\right]
$$

In this case the source term is spatially non-uniform consequently the system can be represented as a heterogeneous heat source heating a two-layered medium. The rear face layer being a homogeneous stratum with no in plane diffusion and constant properties represented by the constant diagonal matrices $\mathbf{C}^{\prime}=C^{\prime} \cdot \mathbf{I}$ and $\mathbf{R}^{\prime}=R^{\prime} \cdot \mathbf{I}$, and the front face being represented by the transfer matrix $\left[\begin{array}{ll}\mathbf{A} & \mathbf{B} \\ \mathbf{C} & \mathbf{D}\end{array}\right]$ detailed in Eq. (4). The flux is now:

$$
\mathbf{Q}=\left[Q l L, \ldots, Q\left(\alpha_{i}, \beta_{j}\right), \ldots\right]^{T}
$$
obtained:

Thus, with similar assumptions, a simple relation analogue to Eq. (7) is

$$
\mathbf{T}(z=2 e, p)=\left(\mathbf{C}^{\prime} \mathbf{R C}\right)^{-1} \mathbf{Q} / p
$$

And by following the same reasoning as in Eq. $(8,9,10)$, it yields:

$$
\left[\frac{\partial T}{\partial t}-a \cdot\left(\frac{\partial^{2} T}{\partial x^{2}}+\frac{\partial^{2} T}{\partial y^{2}}\right)\right](x, y, z=e, t)=\frac{1}{R^{\prime}} \frac{Q(x, y)}{(\rho c \cdot e)^{2}} \cdot t
$$

The term $\frac{1}{R^{\prime}} \frac{Q(x, y)}{(\rho c \cdot e)^{2}}$ inside the previous expression can represent a nonuniform heat flux or a non uniform capacity distribution or contact resistance.

Such simple models are physically non-accurate, but can be used in fast detection algorithms. They are particularly adapted to Total Least Squares algorithms.

\section{Estimation method: The Total Least Squares}

In the case of linear systems such as:

$$
A(x, y, t)=B(x, y, t) \cdot \beta(x, y)
$$

Where $A(x, y, t)$ is the observable at location $(x, y)$ and time $t, \beta(x, y)$ is the parameter vector constant over time and $B(x, y, t)$ contains the elements of the linear model such as derivatives with respect to time or space variables. In the case of the above example, the different terms of Eq (16) are: 


$$
\left\{\begin{array}{l}
A(x, y, t)=\frac{\partial T}{\partial t}(x, y, t) \\
B(x, y, t)=\left[\begin{array}{ll}
t & \frac{\partial^{2} T}{\partial x^{2}}(x, y, t)
\end{array}\right] \\
\beta(x, y)=\left[\begin{array}{c}
\tilde{Q}(x, y) \\
(\rho c \cdot e)^{2} \\
a(x, y)
\end{array}\right]
\end{array}\right.
$$

With notations

$\tilde{Q}(x, y)=\frac{Q}{R(x, y)}$ for thermal bridges; $\tilde{Q}(x, y)=\frac{Q(x, y)}{R^{\prime}}$ for resistive wire

The system (16) is underdetermined so more than one time steps are required to compute $\beta(x, y)$. Indeed if $\beta(x, y)$ is assumed constant over time it stems that:

$$
\mathbf{A}(x, y)=\mathbf{B}(x, y) \cdot \beta(x, y)
$$

With vector notation

$$
\begin{aligned}
& \mathbf{A}(x, y)=\left[\begin{array}{lll}
A\left(x, y, t_{1}\right) & \cdots & A\left(x, y, t_{N}\right)
\end{array}\right]^{T} \\
& \mathbf{B}(x, y)=\left[\begin{array}{lll}
B\left(x, y, t_{1}\right) & \cdots & B\left(x, y, t_{N}\right)
\end{array}\right]^{T}
\end{aligned}
$$

It is demonstrated [12] that a more convenient formulation is:

$$
\left[\begin{array}{ll}
\mathbf{A}(x, y) & \mathbf{B}(x, y)
\end{array}\right] \cdot\left[\begin{array}{ll}
1 & \beta(x, y)
\end{array}\right]^{T}=0
$$

This formulation enables a resolution in the Total Least Squares (TLS) fashion. The TLS estimator is derived from the Ordinary Least Squares (OLS) and is more adequate here because it takes into account the errors in the variables of the linear model [8]. The TLS methodology consists in finding the eigenvector $V_{\text {min }}(x, y)$ to the smallest eigenvalue $\lambda_{\min }(x, y)$ of $\mathbf{J}(x, y)$ with:

$$
\mathbf{J}=\left[\begin{array}{ll}
\mathbf{A}(x, y) & \mathbf{B}(x, y)
\end{array}\right]^{T} \cdot\left[\begin{array}{ll}
\mathbf{A}(x, y) & \mathbf{B}(x, y)
\end{array}\right]
$$

Such as

$$
[1 \quad \beta(x, y)]^{T}=V_{\min }(x, y)
$$


with

$$
\mathbf{J}(x, y)=\left[\begin{array}{ccc}
\sum_{t} t^{2} & -\sum_{t} t \times\left(\frac{\partial^{2} T}{\partial x^{2}}+\frac{\partial^{2} T}{\partial y^{2}}\right) & \sum_{t} t \times \frac{\partial T}{\partial t} \\
s y m & \sum_{t}\left(\frac{\partial^{2} T}{\partial x^{2}}+\frac{\partial^{2} T}{\partial y^{2}}\right)^{2} & \sum_{t} \frac{\partial T}{\partial t} \times\left(\frac{\partial^{2} T}{\partial x^{2}}+\frac{\partial^{2} T}{\partial y^{2}}\right) \\
s y m & s y m & \sum_{t} \frac{\partial T^{2}}{\partial t}
\end{array}\right]
$$

The Least Squares estimator is the maximum likelihood estimator when $\mathbf{B}(x, y, t)$ is known without error [4]. Yet in the case when $T(x, y, t)$ is a temperature measured with an Infrared camera $\frac{\partial T}{\partial t}, \frac{\partial^{2} T}{\partial x^{2}}$, and $\frac{\partial^{2} T}{\partial y^{2}}$ are corrupted by measurement noise that may lead to a bias in the estimation process. For all these reasons, the Total Least Squares offer an improved version of the classical Least Squares estimator [12]. TLS compensate for errors in the variables and present other interesting features: They provide the number of parameters that can be estimated at each location, and may give a confidence measure associated to the estimation. A simple use of the TLS with no prior knowledge of the measurement noise variance is possible thanks to the automatic computation of a threshold using Akaiké's theory [13]. Complementary details about TLS and Akaiké method are given in a parallel paper [14]. Thermophysical parameters are computed using experimental data, mappings are drawn and permit to locate and measure the dimensions of the source terms.

\section{Experimental results}

The aforementioned model was used to implement the TLS estimator on two distinct infrared image sequences. The first application concerns the simultaneous detection of a thermal bridge and the measure of its dimensions in parallel with diffusivity estimation. The second application deals with the detection and measurement of a source term under a thin diffusive plate along with a diffusivity estimation of the plate. Both cases are modelled with a same simplified model. The thermal scene is filmed by a JADE camera (CEDIP) equipped with a $256 \times 256 \mathrm{InSb}$ detector matrix working in the $[1,5 \mu \mathrm{m} ; 5,2 \mu \mathrm{m}]$ range. The spatial resolution of the camera using a lens of focal length $l=25 \cdot 10^{-3} \mathrm{~m}$ is roughly $\Delta x \approx 305 \cdot 10^{-6} \mathrm{~m}$. 


\subsection{Thermal bridge detection}

The experiment presented here corresponds to the device depicted on fig 1 .

Two $7 \cdot 10^{-4} m$ thick steel plates are separated by a thin insulating foam layer and the thermal bridge is a small metallic pillar.
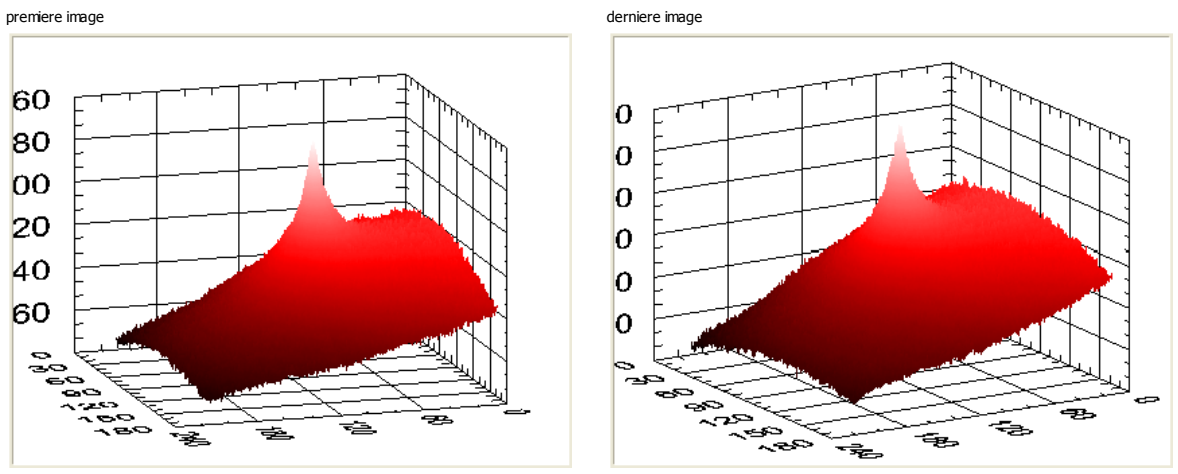

Fig. 3. First and last image of the IR sequence
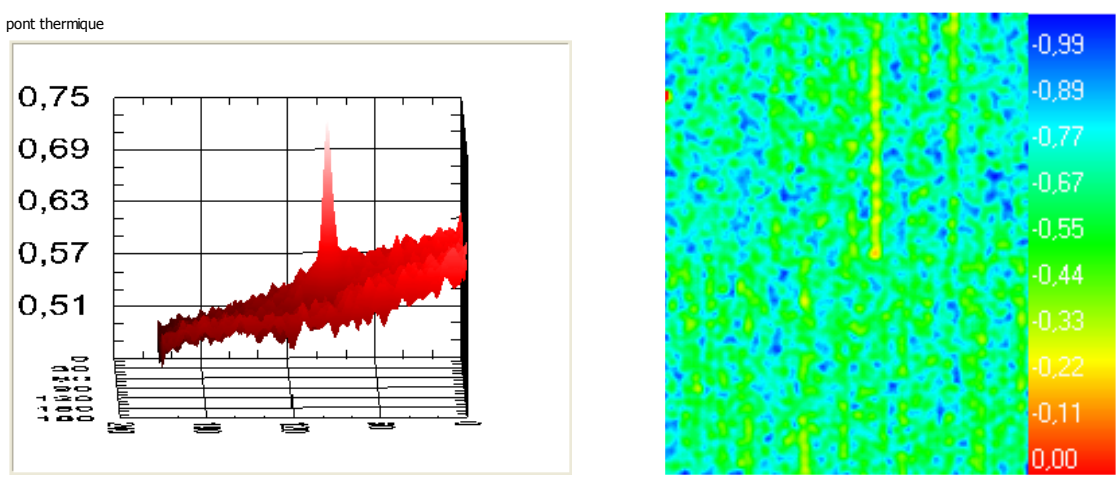

Fig. 4. Location of the thermal bridge, Diffusivity mapping

Thanks to the image processing a mapping with enhanced contrast is obtained. A circle of diameter $d \approx 6$ pixels is measured. Given the spatial calibration of the camera, this corresponds to a $1,8 \cdot 10^{-3} \mathrm{~m}$ diameter. This result is encouraging since the real diameter of the thermal bridge is $2 \cdot 10^{-3} \mathrm{~m}$. The accuracy of the diffusivity mapping is not investigated here, though the fact that the thermal bridge does not appear on the diffusivity mapping is coherent with the simplified model. 


\subsection{Source term detection}

The experiment presented here corresponds to the device depicted on fig 2. A heating wire is fixed under a $7 \cdot 10^{-4} \mathrm{~m}$ thick conductive steel plate.
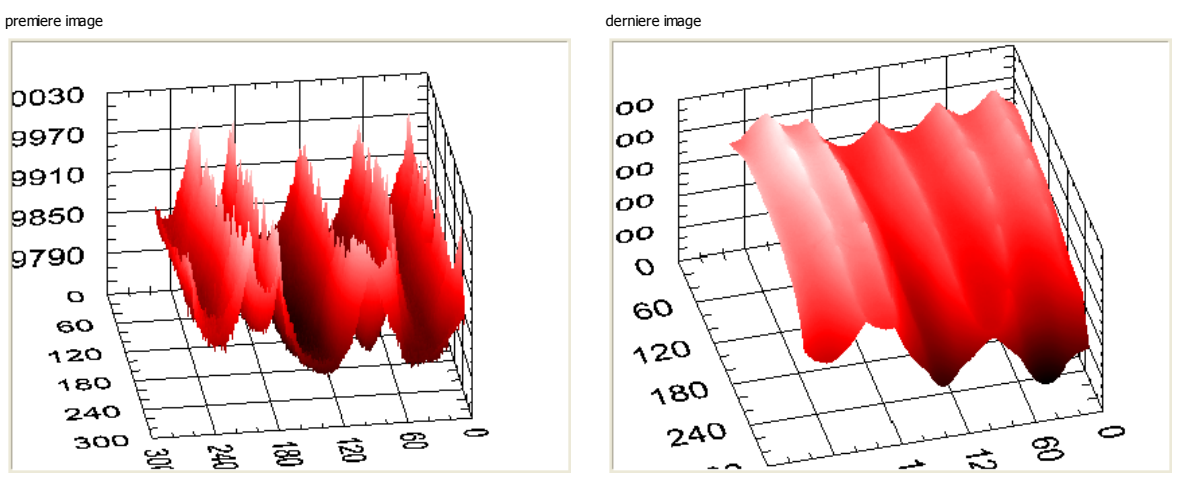

Fig. 5. First and last image of the IR sequence
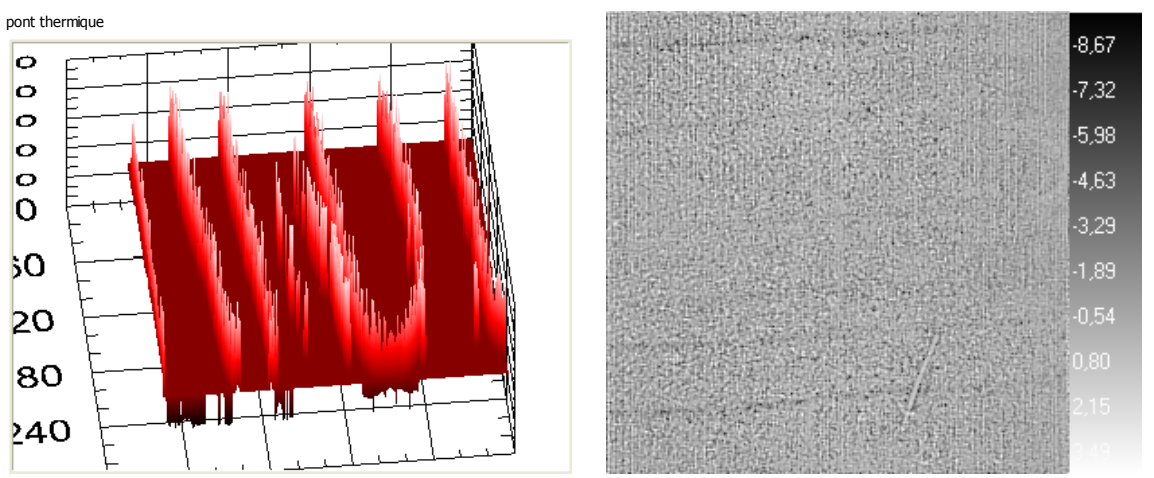

Fig. 6. Location of the source terms, Diffusivity mapping

Again here the contrast is enhanced and the source term width and position is clearly displayed. The measured width is equivalent to $0,5 \cdot 10^{-3} \mathrm{~m}$ diameter as compared to the real width which is $0,35 \cdot 10^{-3} \mathrm{~m}$. Yet the detected source term is only 2 to 3 pixels large, at this scale the approximate spatial calibration of the camera is clearly a limit to the fine measurement of the wire diameter.

\section{$5 \quad$ Conclusions and perspectives}

Simple models were developed to describe thermal transfers in 3D multilayered media in order to obtain direct expressions with linear differential terms versus time or space. These models allowed applying fast Total Least Squares 


\section{http://dx.doi.org/10.21611/qirt.2006.059}

algorithms to inverse the temperature field. The main advantages of such algorithms are to allow a nodal approach instead of the inversion of very large linear systems. It can be useful in the case of small sized thermal bridges Even if the approximations are not accurate, such algorithms can be used as primary approach in order to enhance the defect detection.

\section{REFERENCES}

[1] D. Balageas, P. Delpech, D. Boscher, A. Deom, New developments in stimulated infrared thermography applied to non destructive evaluation of laminates, Review on Progress in Quantitative Non-Destructive Testing, ED Thompson and Chimienti. Plenum Press, New York, 1991, Vol 10 A, pp 10731081

[2] J.-C. Batsale, J.-L. Battaglia, O. Fudym, Autoregressive algorithms and spatially random flash excitation for 2D non destructive evaluation with infrared cameras, QIRT journal, Vol1-N¹-2004, p. 5-20

[3] Fudym O., Santander R., Batsale J.-C., Battaglia J.-L., "Thermophysical properties mapping in semi infinite longitudinally cracked plates by temperature image processing", Inverse problems, Design and Optimization Symposium, Rio de Janeiro, Brazil, 2004

[4] D. Mourand, J.C. Batsale, J.L. Battaglia, Infrared image processing for the evaluation of thermal bridges in high performance insulating systems, Image Analysis \& Stereology, N²0 (2) supl. 1 (2001), 498-503.

[5] Mourand D., Batsale J.C. et Gounot J. - "New sequential method to process noisy temperature response from flash experiment measured by infrared camera" - Review of Scientific Instrument, Vol 69, n³, 1998, p. 1437-1441.

[6] Mourand D., Batsale J.C., Sequential method for thermal diffusivities discrimination by infrared thermography, High Temperatures-High Pressures, Vol 33, 2001, p127-134.

[7] B. Jähne, H.W. Haussecker, H. Scharr, H. Spies, D. Schmundt, U. Schurr: Study of Dynamical Processes with Tensor-Based Spatiotemporal Image Processing Techniques, ECCV (2) 1998: 322-336

[8] S. Van Huffel, J. Vandewalle, The Total Least Squares Problem Computational Aspects and Analysis, Society for Industrial and Applied Mathematics Philadelphia (1991)

[9] Maillet D., Batsale J.C, Degiovanni A., André S., Moyne C., Thermal Quadrupoles, solving the heat equation through integral transforms (J. Wiley and sons 2000)

[10] J.C. Batsale, D. Maillet, A. Degiovanni, Extension de la méthode des quadripoles thermiques à l'aide de transformations intégrales-Application au défaut plan bidimensionnel, Int J. Heat Mass Transfer, 1994, Vol 37, n1, pp111127.

[11] A. Bendada, D. Maillet, J.C. Batsale, A. Degiovanni, Reconstruction of a nonuniform interface thermal resistance by inverse conduction, Inverse Problems in Engineering, Vol 6, 1998, p. 79-123.

[12] Beck JV and Arnold K. J., Parameter estimation in engineering and science, John Wiley and Sons, NY, 1977

[13] Akaike H., "A new look at the statistical model identification", IEEE Transactions on Automatic Controls, Vol AC-19, December 1974: pp716-723

[14] Bamford M., Batsale JC., Rungoat D., Fudym O., Two dimensional velocity and diffusion mapping in the case of three-dimensional transient diffusion: "Flash method and infrared image sequence analysis, QIRT 2006. 